University of Wollongong

Research Online

Faculty of Engineering and Information

Faculty of Engineering and Information

Sciences - Papers: Part A

Sciences

$1-1-2016$

Coupled nonlinear dynamics of geometrically imperfect shear deformable extensible microbeams

Mergen H. Ghayesh

University of Wollongong, mergen@uow.edu.au

Hamed Farokhi

McGill University

Follow this and additional works at: https://ro.uow.edu.au/eispapers

Part of the Engineering Commons, and the Science and Technology Studies Commons

Research Online is the open access institutional repository for the University of Wollongong. For further information contact the UOW Library: research-pubs@uow.edu.au 


\title{
Coupled nonlinear dynamics of geometrically imperfect shear deformable extensible microbeams
}

\begin{abstract}
This paper aims at analyzing the coupled nonlinear dynamical behavior of geometrically imperfect shear deformable extensible microbeams based on the third-order shear deformation and modified couple stress theories. Using Hamilton's principle and taking into account extensibility, the three nonlinear coupled continuous expressions are obtained for an initially slightly curved (i.e., a geometrically imperfect) microbeam, describing the longitudinal, transverse, and rotational motions. A high-dimensional Galerkin scheme is employed, together with an assumed-mode technique, in order to truncate the continuous system with an infinite number of degrees of freedom into a discretized model with sufficient degrees of freedom. This high-dimensional discretized model is solved by means of the pseudo-arclength continuation technique for the system at the primary resonance, and also by direct time-integration to characterize the dynamic response at a fixed forcing amplitude and frequency; stability analysis is conducted via the Floquet theory. Apart from analyzing the nonlinear resonant response, the linear natural frequencies are obtained via an eigenvalue analysis. Results are shown through frequency-response curves, force-response curves, time traces, phase-plane portraits, and fast Fourier transforms (FFTs). The effect of taking into account the length-scale parameter on the coupled nonlinear dynamic response of the system is also highlighted.
\end{abstract}

\section{Keywords}

extensible, deformable, shear, microbeams, imperfect, coupled, geometrically, dynamics, nonlinear

\section{Disciplines}

Engineering | Science and Technology Studies

\section{Publication Details}

Ghayesh, M. H. \& Farokhi, H. (2016). Coupled nonlinear dynamics of geometrically imperfect shear deformable extensible microbeams. Journal of Computational and Nonlinear Dynamics, 11 (4), 041001-1-041001-10. 


\author{
Mergen H. Ghayesh ${ }^{1}$ \\ School of Mechanical, Materials \\ and Mechatronic Engineering, \\ University of Wollongong, \\ Wollongong NSW 2522, Australia \\ e-mail: mergen@uow.edu.au \\ Hamed Farokhi \\ Department of Mechanical Engineering, \\ McGill University, \\ Montreal, QC H3A OC3, Canada \\ e-mail: hamed.farokhi@mail.mcgill.ca
}

\section{Coupled Nonlinear Dynamics of Geometrically Imperfect Shear Deformable Extensible Microbeams}

This paper aims at analyzing the coupled nonlinear dynamical behavior of geometrically imperfect shear deformable extensible microbeams based on the third-order shear deformation and modified couple stress theories. Using Hamilton's principle and taking into account extensibility, the three nonlinear coupled continuous expressions are obtained for an initially slightly curved (i.e., a geometrically imperfect) microbeam, describing the longitudinal, transverse, and rotational motions. A high-dimensional Galerkin scheme is employed, together with an assumed-mode technique, in order to truncate the continuous system with an infinite number of degrees of freedom into a discretized model with sufficient degrees of freedom. This high-dimensional discretized model is solved by means of the pseudo-arclength continuation technique for the system at the primary resonance, and also by direct time-integration to characterize the dynamic response at a fixed forcing amplitude and frequency; stability analysis is conducted via the Floquet theory. Apart from analyzing the nonlinear resonant response, the linear natural frequencies are obtained via an eigenvalue analysis. Results are shown through frequency-response curves, force-response curves, time traces, phase-plane portraits, and fast Fourier transforms (FFTs). The effect of taking into account the length-scale parameter on the coupled nonlinear dynamic response of the system is also highlighted.

[DOI: $10.1115 / 1.4031288]$

Keywords: coupled nonlinear dynamics, third-order shear deformation theory, modified coupled stress theory, initial imperfection

\section{Introduction}

Continuous microsystems are present in many engineering devices and micromachines [1,2], for example, in micro energy harvesters, airbag accelerometers, biosensors, biomechanical organs, microactuators, microswitches, MEMS resonators, and vibration shock sensors [3-8]; due to their widespread application, many researchers have investigated the dynamical behavior of these systems in recent years. An important issue in the theoretical modeling of microscale continuous systems is to use a continuum theory which is capable of capturing small-size effects; the dependence on size was first found out experimentally [9-11]. Between the theories capable of taking into account size effects, the modified couple stress theory [12] is employed in this paper. The literature concerning the static and dynamic behaviors of microbeams can be classified mainly into two general groups in terms of the models being considered. The first group analyzed the statics or dynamics of microbeams based on either the EulerBernoulli or Timoshenko beam theory. The second class employed higher-order shear deformation theories.

Starting the literature review of the first group, the natural frequencies of microbeams were obtained by Kong et al. [13] and Ma et al. [14], based on the Euler-Bernoulli and Timoshenko beam theories, respectively. Asghari et al. [15] and Wang et al. [16] employed the modified couple stress theory and a strain gradient elasticity theory, respectively, in order to model Timoshenko microbeams and study their size-dependent behavior. Ansari et al. [17-19] analyzed the size-dependent bending and thermal

\section{${ }^{1}$ Corresponding author.}

Contributed by the Design Engineering Division of ASME for publication in the Journal of Computational and Nonlinear Dynamics. Manuscript received December 8, 2014; final manuscript received August 10, 2015; published online November 13, 2015. Assoc. Editor: Daniel J. Segalman. buckling and postbuckling of functionally graded Timoshenko microbeams based on a strain gradient elasticity theory. Nateghi et al. [20] contributed to the field by examining the buckling response of functionally graded microbeams based on the modified couple stress theory. Akgöz and Civalek [21] investigated the buckling behavior of an axially forced microbeam employing both the modified couple stress and classical couple stress theories. Ouakad and Younis [22] examined the dynamic snap-through motion of an electrically actuated initially curved microbeam and investigated the possibility of using the snap-through motion for filtering applications. Farokhi et al. [23] continued the investigations by examining the nonlinear dynamics of microbeams beyond buckling, thorough use of the modified couple stress theory. Ramezani [24] and Asghari et al. [25] analyzed the nonlinear free dynamics of Timoshenko microbeams by means of the method of multiple time scale based on strain gradient elasticity and modified couple stress theories, respectively.

The literature regarding the second class is not large. Addressing the linear aspects of the problem, for example, Salamat-talab et al. [26] examined the linear statics and dynamics of a functionally graded microbeam employing a third-order shear deformation and the modified couple stress theories in the modeling. Şimşek and Reddy [27,28] investigated the linear bending, buckling, and oscillations of functionally graded microbeams employing a highorder shear deformation theory based on the modified couple stress theory. Sahmani and Ansari [29] examined the linear buckling response of functionally graded microbeams under thermal loadings, based on a third-order shear deformation theory. Zhang et al. [30] employed a strain gradient elasticity theory in order to develop a model for a functionally graded microbeam via a thirdorder shear deformation theory.

All of the studies reviewed in the previous paragraph were concerned with linear modeling and analysis of the dynamics of 
higher-order shear deformable microbeams. Apart from this, in all of those studies, the microbeam is assumed to be perfectly straight; however, it is highly possible to manufacture an initially curved (i.e., geometrically imperfect) microbeam due to improper manufacturing process. Also, in some applications, such as in microswitches, the deformable electrode is manufactured initially curved intentionally to display a snap-through motion for on/off states. As such, the current paper examines the nonlinear forced dynamics of initially imperfect third-order shear deformable extensible microbeams based on the modified couple stress theory. More specifically: (1) a third-order shear deformable microbeam with a geometric imperfection is considered - this theory allows for accurate prediction of the shear force (which becomes considerable for thick systems) throughout the thickness; (2) the strain energy is obtained in terms of the displacement field based on the modified couple stress theory [23,31-35]; (3) the kinetic energy of the system and the external works are determined in terms of the displacement field; (4) a variational technique based on Hamilton's principle is employed to derive the continuous models governing the longitudinal, transverse, and rotational motions; (5) a high-dimensional Galerkin scheme is used to transform the continuous system to a discretized model; (6) the linear natural frequencies are obtained by means of an eigenvalue analysis; (7) a continuation technique and a direct time-integration method are employed to obtain the nonlinear response numerically; (8) the effect of system parameters, including the initial curvature and the length-scale parameter, on the system response is highlighted; (9) results are shown via frequency-responses, force-responses, time traces, phase-plane diagrams, and FFTs.

\section{Model Development}

The schematic representation of the system under consideration is shown in Fig. 1, illustrating a simply supported third-order shear deformable microbeam with immovable ends (which induces extensibility). $L, \rho, E, A$, and $I$ denote the length, mass density, Young's modulus, cross-sectional area, and second area moment of the cross section of the microbeam, respectively. The displacements in the longitudinal and transverse directions are represented by $u(x, t)$ and $w(x, t)$, respectively; $\phi(x, t)$ denotes the rotation of the transverse normal. The microbeam is excited harmonically in the transverse direction by a distributed force per unit length, $F(x) \cos (\omega t)$. The initial curvature (geometric imperfection) is modeled in the form of a function of $x$, denoted by $w_{0}(x)$.

The potential energy of the system can be expressed, based on the modified couple stress theory, as

$$
U=\frac{1}{2} \int_{V}(\boldsymbol{\sigma}: \boldsymbol{\varepsilon}+\mathbf{m}: \boldsymbol{\chi}) d v
$$

where $\boldsymbol{\sigma}$ and $\boldsymbol{\varepsilon}$ represent the stress and strain tensors, respectively; $\mathbf{m}$ and $\chi$ represent the deviatoric part of the symmetric couple stress and the symmetric curvature tensors, respectively.

The displacement field for a generic point at a distance $z$ form the centerline of the microbeam is shown by vector $\mathbf{u}$, given by

$$
\mathbf{u}=\left[\begin{array}{l}
u(x, t)+z \phi(x, t)-\frac{4}{3 h^{2}} z^{3}\left(\phi(x, t)+\frac{\partial w(x, t)}{\partial x}\right) \\
0 \\
w(x, t)+w_{0}(x)
\end{array}\right]
$$

in which $h$ represents the thickness of the microbeam and $w_{0}(x)$ denotes the initial curvature associated with zero initial stresses.

Knowing that $\boldsymbol{\theta}=(1 / 2) \operatorname{curl}(\mathbf{u})$, the symmetric curvature tensor $\chi$ can be formulated as a function of the rotation vector, $\boldsymbol{\theta}$, as

$$
\chi=\frac{1}{2}\left(\nabla \boldsymbol{\theta}+(\nabla \boldsymbol{\theta})^{\mathrm{T}}\right)
$$

which gives

$$
\begin{aligned}
& \chi_{x y}=\chi_{y x}=\frac{1}{4}\left[\frac{\partial \phi}{\partial x}-\frac{\partial^{2} w}{\partial x^{2}}-\frac{4 z^{2}}{h^{2}}\left(\frac{\partial \phi}{\partial x}+\frac{\partial^{2} w}{\partial x^{2}}\right)\right] \\
& \chi_{y z}=\chi_{z y}=-\frac{2 z}{h^{2}}\left(\phi+\frac{\partial w}{\partial x}\right)
\end{aligned}
$$

Based on Eq. (4), the application of the following constitutive relation, valid for isotropic linear elastic materials:

$$
m_{i j}=2 \mu l^{2} \chi_{i j}
$$

gives

$$
\begin{aligned}
& m_{x y}=m_{y x}=\frac{1}{2} \mu l^{2}\left[\frac{\partial \phi}{\partial x}-\frac{\partial^{2} w}{\partial x^{2}}-\frac{4 z^{2}}{h^{2}}\left(\frac{\partial \phi}{\partial x}+\frac{\partial^{2} w}{\partial x^{2}}\right)\right] \\
& m_{y z}=m_{z y}=-4 \mu l^{2} \frac{z}{h^{2}}\left(\phi+\frac{\partial w}{\partial x}\right)
\end{aligned}
$$

where $l$ is the material length-scale parameter and $\mu$ is the shear modulus.

The strain tensor, according to Eq. (2), can be expressed as

$$
\begin{aligned}
& \varepsilon_{x x}=\frac{\partial u}{\partial x}+\frac{1}{2}\left(\frac{\partial w}{\partial x}\right)^{2}+\frac{\partial w}{\partial x} \frac{\mathrm{d} w_{0}}{\mathrm{~d} x}+z \frac{\partial \phi}{\partial x}-\frac{4 z^{3}}{3 h^{2}}\left(\frac{\partial \phi}{\partial x}+\frac{\partial^{2} w}{\partial x^{2}}\right) \\
& \varepsilon_{x z}=\varepsilon_{z x}=\frac{1}{2}\left(1-\frac{4 z^{2}}{h^{2}}\right)\left(\phi+\frac{\partial w}{\partial x}\right)
\end{aligned}
$$

The nonzero components of the stress tensor can then be formulated as

$$
\begin{aligned}
& \sigma_{x x}=E\left[\frac{\partial u}{\partial x}+\frac{1}{2}\left(\frac{\partial w}{\partial x}\right)^{2}+\frac{\partial w}{\partial x} \frac{\mathrm{d} w_{0}}{\mathrm{~d} x}+z \frac{\partial \phi}{\partial x}-\frac{4 z^{3}}{3 h^{2}}\left(\frac{\partial \phi}{\partial x}+\frac{\partial^{2} w}{\partial x^{2}}\right)\right] \\
& \sigma_{x z}=\sigma_{z x}=\mu\left(1-\frac{4 z^{2}}{h^{2}}\right)\left(\phi+\frac{\partial w}{\partial x}\right)
\end{aligned}
$$

Substitution of Eqs. (4), (6), (7), and (8) into Eq. (1) results in

$$
\begin{aligned}
U= & E I \int_{0}^{L}\left[\frac{34}{105}\left(\frac{\partial \phi}{\partial x}\right)^{2}+\frac{1}{42}\left(\frac{\partial^{2} w}{\partial x^{2}}\right)^{2}-\frac{16}{105} \frac{\partial^{2} w}{\partial x^{2}} \frac{\partial \phi}{\partial x}\right] \mathrm{d} x \\
& +\frac{1}{2} E A \int_{0}^{L}\left[\frac{\partial u}{\partial x}+\frac{1}{2}\left(\frac{\partial w}{\partial x}\right)^{2}+\frac{\partial w}{\partial x} \frac{\mathrm{d} w_{0}}{\mathrm{~d} x}\right]^{2} \mathrm{~d} x \\
& +\frac{4}{15} \mu A \int_{0}^{L}\left(\frac{\partial w}{\partial x}+\phi\right)^{2} \mathrm{~d} x+\mu A l^{2} \int_{0}^{L}\left[\frac{1}{15}\left(\frac{\partial \phi}{\partial x}\right)^{2}\right. \\
& \left.+\frac{7}{30}\left(\frac{\partial^{2} w}{\partial x^{2}}\right)^{2}-\frac{1}{5} \frac{\partial^{2} w}{\partial x^{2}} \frac{\partial \phi}{\partial x}+\frac{2}{3 h^{2}}\left(\frac{\partial w}{\partial x}+\phi\right)^{2}\right] \mathrm{d} x
\end{aligned}
$$

The variation of the works due to the external excitation and damping (with a coefficient $c_{\mathrm{d}}$ ) can be formulated as

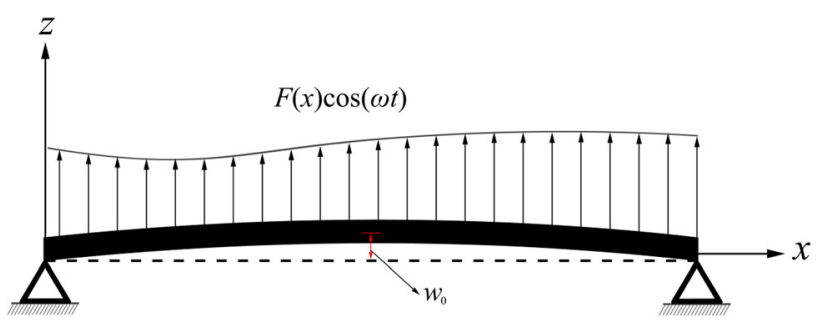

Fig. 1 Schematic representation of an initially curved shear deformable extensible microbeam 


$$
\begin{gathered}
\delta W_{F}=\int_{0}^{L} F(x) \cos (\omega t) \delta w \mathrm{~d} x \\
\delta W_{D}=-c_{d} \int_{0}^{L}\left(\frac{\partial u}{\partial t} \delta u+\frac{\partial w}{\partial t} \delta w+h^{2} \frac{\partial \phi}{\partial t} \delta \phi\right) \mathrm{d} x
\end{gathered}
$$

The kinetic energy of the microbeam can be formulated as

$$
\begin{aligned}
T= & \rho A \int_{0}^{L}\left[\frac{1}{2}\left(\frac{\partial u}{\partial t}\right)^{2}+\frac{1}{2}\left(\frac{\partial w}{\partial t}\right)^{2}\right] \mathrm{d} x \\
& +\rho I \int_{0}^{L}\left[\frac{34}{105}\left(\frac{\partial \phi}{\partial t}\right)^{2}+\frac{1}{42}\left(\frac{\partial^{2} w}{\partial x \partial t}\right)^{2}-\frac{16}{105} \frac{\partial^{2} w}{\partial x \partial t} \frac{\partial \phi}{\partial t}\right] \mathrm{d} x
\end{aligned}
$$

Substituting Eqs. (9)-(12) into generalized Hamilton's principle given by [36]

$$
\int_{t_{1}}^{t_{2}}(\delta T-\delta U) \mathrm{d} t+\int_{t_{1}}^{t_{2}}\left(\delta W_{F}+\delta W_{D}\right) \mathrm{d} t=0
$$

yields the following coupled nonlinear partial differential equations governing the motion of the system in the longitudinal and transverse directions as well as the rotation, respectively

$$
\begin{aligned}
& \rho A \frac{\partial^{2} u}{\partial t^{2}}-E A \frac{\partial}{\partial x}\left(\frac{\partial u}{\partial x}+\frac{1}{2}\left(\frac{\partial w}{\partial x}\right)^{2}+\frac{\partial w}{\partial x} \frac{\mathrm{d} w_{0}}{\mathrm{~d} x}\right)+c_{d} \frac{\partial u}{\partial t}=0 \\
& \rho A \frac{\partial^{2} w}{\partial t^{2}}+\rho I\left(\frac{16}{105} \frac{\partial^{3} \phi}{\partial x \partial t^{2}}-\frac{1}{21} \frac{\partial^{4} w}{\partial x^{2} \partial t^{2}}\right) \\
& \quad-E A \frac{\partial}{\partial x}\left\{\left(\frac{\partial w}{\partial x}+\frac{\mathrm{d} w_{0}}{\mathrm{~d} x}\right)\left[\frac{\partial u}{\partial x}+\frac{1}{2}\left(\frac{\partial w}{\partial x}\right)^{2}+\frac{\partial w}{\partial x} \frac{\mathrm{d} w_{0}}{\mathrm{~d} x}\right]\right\} \\
& +E I\left(\frac{1}{21} \frac{\partial^{4} w}{\partial x^{4}}-\frac{16}{105} \frac{\partial^{3} \phi}{\partial x^{3}}\right)-\frac{8}{15} \mu A\left(\frac{\partial^{2} w}{\partial x^{2}}+\frac{\partial \phi}{\partial x}\right) \\
& \quad+\mu A l^{2}\left[\frac{7}{15} \frac{\partial^{4} w}{\partial x^{4}}-\frac{1}{5} \frac{\partial^{3} \phi}{\partial x^{3}}-\frac{4}{3 h^{2}}\left(\frac{\partial^{2} w}{\partial x^{2}}+\frac{\partial \phi}{\partial x}\right)\right] \\
& \quad-f_{1} \cos (\omega t)+c_{d} \frac{\partial w}{\partial t}=0, \\
& \rho I\left(\frac{68}{105} \frac{\partial^{2} \phi}{\partial t^{2}}-\frac{16}{105} \frac{\partial^{3} w}{\partial x \partial t^{2}}\right)+E I\left(\frac{16}{105} \frac{\partial^{3} w}{\partial x^{3}}-\frac{68}{105} \frac{\partial^{2} \phi}{\partial x^{2}}\right) \\
& +\frac{8}{15} \mu A\left(\frac{\partial w}{\partial x}+\phi\right)+\mu A l^{2}\left[\frac{1}{5} \frac{\partial^{3} w}{\partial x^{3}}-\frac{2}{15} \frac{\partial^{2} \phi}{\partial x^{2}}+\frac{4}{3 h^{2}}\left(\frac{\partial w}{\partial x}+\phi\right)\right] \\
& +c_{d} h^{2} \frac{\partial \phi}{\partial t}=0
\end{aligned}
$$

\section{Galerkin's Scheme, Discretization, and Solution Methods}

Equations (14)-(16) represent the equations of motion of a continuous model with infinite degrees of freedom; this system is converted into a discretized model via application of the Galerkin scheme. As such, the displacements are defined by the following approximate series expansions:

$$
\begin{aligned}
& w(x, t)=\sum_{k=1}^{M} \varphi_{k}(x) q_{k}(t) \\
& \phi(x, t)=\sum_{k=1}^{N} \psi_{k}(x) p_{k}(t) \\
& u(x, t)=\sum_{k=1}^{Q} \varphi_{k}(x) r_{k}(t)
\end{aligned}
$$

where $\varphi_{k}$ denotes the $k$ th eigenfunction for the transverse motion of a linear hinged-hinged beam and $\psi_{k}=\varphi^{\prime}{ }_{k} /(k \pi) ; q_{k}(\mathrm{t}), p_{k}(\mathrm{t})$, and $r_{k}(\mathrm{t})$ represent the $k$ th generalized coordinate for the transverse, rotational, and longitudinal motions, respectively.

Assuming $w_{0}(x)=A_{0} \varphi_{1}(x)$ and $F(x)=f_{1}$, substituting Eqs. (17)-(19) into Eqs. (14)-(16), multiplying both sides of the resultant equation by the corresponding eigenfunctions, and integrating over the spatial domain result in a set of second-order nonlinear ordinary differential equations in the form of

$$
\mathbf{M} \ddot{\mathbf{q}}+\mathbf{C} \dot{\mathbf{q}}+\mathbf{K}_{\mathbf{1}} \mathbf{q}+\mathbf{K}_{\mathbf{2}}(\mathbf{q}) \mathbf{q}+\mathbf{K}_{\mathbf{3}}(\mathbf{q}, \mathbf{q}) \mathbf{q}=\mathbf{f} \cos (\omega t)
$$

where $\mathbf{K}_{1}, \mathbf{K}_{2}$, and $\mathbf{K}_{3}$ denote the linear, quadratic, and cubic stiffness matrices, respectively; $\mathbf{C}$ is the damping matrix; $\mathbf{M}$ is the nondiagonal mass matrix; $\mathbf{f}$ represents the forcing amplitude vector.

Equation (20) represents a set of $(M+N+Q)$ second-order nonlinear ordinary differential equations; in order to be able to solve these equations numerically, this set should be transformed into a set of $2(M+N+Q)$ first-order nonlinear ordinary differential equations. However, this procedure cannot be performed directly due to the inertial coupling between equations which is the result of the nondiagonal mass matrix. The inertial coupling can be solved by diagonalizing the mass matrix, which is done by premultiplying Eq. (20) by $\mathbf{M}^{-1}$, resulting in

$$
\begin{aligned}
\mathbf{I} \ddot{\mathbf{q}} & +\mathbf{M}^{-1} \mathbf{C} \dot{\mathbf{q}}+\mathbf{M}^{-1} \mathbf{K}_{\mathbf{1}} \mathbf{q}+\mathbf{M}^{-1} \mathbf{K}_{\mathbf{2}}(\mathbf{q}) \mathbf{q}+\mathbf{M}^{-1} \mathbf{K}_{\mathbf{3}}(\mathbf{q}, \mathbf{q}) \mathbf{q} \\
& =\mathbf{M}^{-1} \mathbf{f} \cos (\omega t)
\end{aligned}
$$

Equation (21) is transformed into a new set of $2(M+N+Q)$ firstorder nonlinear ordinary differential equations through use of a change of variables. The new set of equations is solved by means of: (1) an eigenvalue analysis for the determination of the linear natural frequencies of the system; (2) the pseudo-arclength continuation technique as well as the Floquet theory for the analysis of nonlinear resonant responses and determining stability and bifurcations; (3) a direct time-integration technique, based on the variable step-size Rosenbrock method. These numerical techniques help us in constructing frequency-responses, force-responses, time histories, phase-plane portraits, and FFTs. In the present study, $M=N=Q=16$ is selected; however, due to the symmetric configuration of the system and external excitation, only the symmetric modes contribute to the response of the system; hence, a 24-degree-of-freedom system is examined, which ensures converged and reliable results. This number of modes results in 48 first-order nonlinear ordinary differential equations with coupled terms; solving such a high-dimensional system of equations numerically necessitates developing accurate codes with optimized run-time.

\section{Coupled Nonlinear Dynamical Behavior of the System}

This section examines the coupled nonlinear dynamical response, particularly by constructing frequency-responses, force-responses, time histories, phase-plane portraits, and FFTs. As a test-case, calculations have been performed for a microbeam made of epoxy with: $l=17.6 \mu \mathrm{m}, h=3.65 l, L=200 h, b=6 h$, $E=1.44 \mathrm{GPa}, \mu=521.7 \mathrm{MPa}$, and $\rho=1220 \mathrm{~kg} / \mathrm{m}^{3}$ ( $b$ denotes the width of the microbeam). The following dimensionless parameters are used throughout the numerical simulations:

$$
\begin{aligned}
& u^{*}=\frac{u}{h}, \quad w^{*}=\frac{w}{h}, \quad A_{0}^{*}=\frac{A_{0}}{h}, \quad \Omega=\omega \sqrt{\frac{\rho A L^{4}}{E I}}, \quad t^{*}=t \sqrt{\frac{E I}{\rho A L^{4}}} \\
& f_{1}^{*}=\frac{f_{1} L^{4}}{E I h}, \quad c_{d}^{*}=\frac{c_{d} L^{4}}{E I} \sqrt{\frac{E I}{\rho A L^{4}}}, \quad \omega_{1}^{*}=\omega_{1} \sqrt{\frac{\rho A L^{4}}{E I}}
\end{aligned}
$$


In what follows, only the dimensionless parameters are used and reported and the asterisk notation is dropped for briefness.

Figure 2 shows the nonlinear responses of the microbeam at the primary resonance; the following parameters are selected: $f_{1}=1.15$ and $A_{0}=0.2$, giving $\omega_{1}=12.3518$, where $\omega_{1}$ is the first linear natural frequency of the transverse motion; moreover, the modal damping ratio is set to $\zeta=0.009$ for all generalized coordinate throughout the numerical simulation. As seen in the figure, the system displays a hardening-type nonlinear behavior with two limit-point bifurcations at $\Omega=1.0935 \omega_{1}$ and $\Omega=1.0305 \omega_{1}$. A comparison between subfigures $(a)$ and $(c)$ reveals that the longitudinal displacement is much smaller than the transverse displacement; however, this does not mean that the effect of the longitudinal displacement is negligible. The reason is that the nonlinear terms in the equations of motion involving $u$ (i.e., the longitudinal motion) are quadratic which makes them comparable to cubic nonlinear terms involving $w$ (i.e., the transverse motion). Moreover, canceling $u$ in the modeling changes the $w$ response. The details of the system dynamics at $\Omega=1.0290 \omega_{1}$ are depicted in Fig. 3, through a time trace, phase-plane portrait, and FFT of the $q_{1}$ motion. The comparison between the frequency-response curves of the system obtained via the modified couple stress and classical continuum theories are displayed in Fig. 4; it is seen that the maximum amplitude of the oscillation predicted by the classical continuum theory is fairly larger than that predicted by the modified couple stress theory. In addition, the modified couple stress theory predicts a weaker nonlinear hardening behavior.

Increasing the forcing amplitude and the amplitude of the initial imperfection to $f_{1}=2.85$ and $A_{0}=0.4$, respectively, a new set of figures is obtained and plotted in Fig. 5. As seen, due to increased amplitude of the initial imperfection, the nonlinear resonant response of the system is a softening-hardening type, showing the simultaneous effect of the quadratic and cubic nonlinear terms in the equation of motion-such a behavior has already been reported by researchers for macrosystems, for instance by Benedettini and Rega [37,38] for suspended cables under primary and subharmonic excitations, showing a qualitative behavior similar to that of the system examined here. The initial softening and the followed hardening behaviors are due to the presence of quadratic and cubic nonlinear terms, respectively, in the equations of motion. Subfigure 5(a) shows that, theoretically, as the excitation frequency is increased from $\Omega=0.8200 \omega_{1}$, the periodic response amplitude increases accordingly until point $\operatorname{LP}_{1}\left(\Omega=0.9398 \omega_{1}\right)$ is hit, corresponding to the first limit-point bifurcation and hence instability. Stability is regained at point $\operatorname{LP}_{2}\left(\Omega=0.9176 \omega_{1}\right)$ via the second limit-point bifurcation. The amplitude of the stable response increases with the excitation frequency until hitting point $\mathrm{LP}_{3}\left(\Omega=1.0434 \omega_{1}\right)$; at this point, the third limit-point bifurcation occurs which leads the system to have an unstable solution. By decreasing $\Omega$, the amplitude of this new unstable response decreases accordingly until point $\operatorname{LP}_{4}\left(\Omega=0.9453 \omega_{1}\right)$ is reached, where the stability is regained once again via the fourth limitpoint bifurcation; the response amplitude decreases with $\Omega$ thereafter. It should be noted that, as seen in subfigure $(c)$, although the amplitude of the longitudinal is small compared to transverse and rotational motion, it affects their motion by introducing quadratic nonlinearities, hence strengthening the softening behavior and weakening the hardening one.

A comparison is made in Fig. 6 between the nonlinear resonant response plots (i.e., frequency-response curves) of the microbeam modeled by means of the modified couple stress and classical continuum theories. For the system modeled based on the modified couple stress theory (see subfigure $(a)$ ), both the softening- and hardening-type behaviors are weaker. Moreover, the peak amplitude for the case of the modified couple stress theory is smaller than that of the classical continuum theory.

Figure 7 addresses the effect of the amplitude of the initial imperfection, $A_{0}$, on the nonlinear resonant response of the system, shown by frequency-response curves here. As seen in subfigure $(a)$, due to increased $A_{0}$, the initial softening behavior (a)

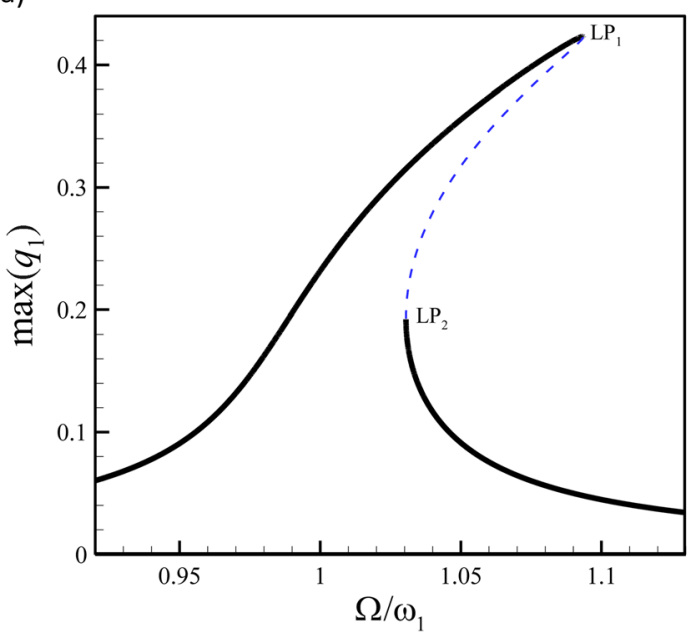

(b)

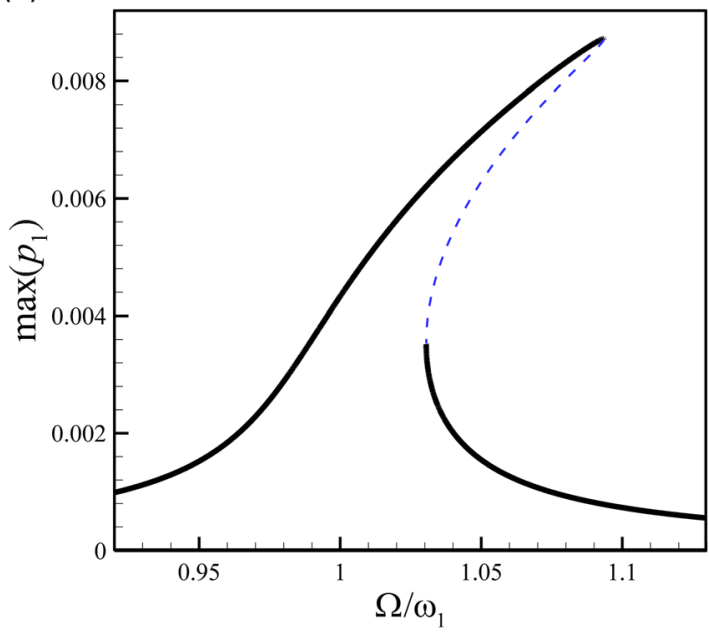

(c)

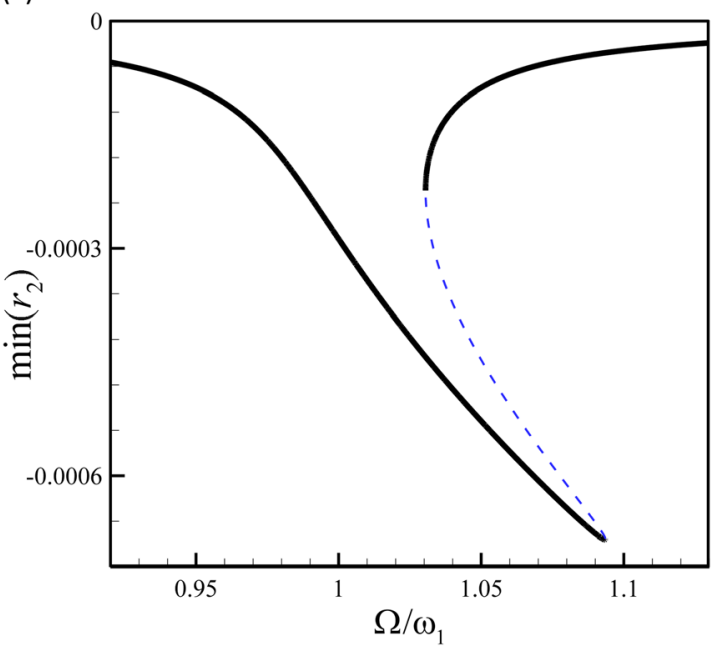

Fig. 2 Frequency-response curves of the system: (a) and (b) the first generalized coordinate of the transverse motion and rotation, respectively; (c) the second generalized coordinate of the longitudinal motion. Solid and dashed lines represent the stable and unstable solutions, respectively; $f_{1}=1.15$ and $A_{0}=0.2$

becomes stronger, and the hardening region becomes smaller; it is seen that the hardening behavior almost vanishes for the case with $A_{0}=0.5$. Moreover, the peak-amplitude becomes smaller when $A_{0}$ is increased. 
(a)

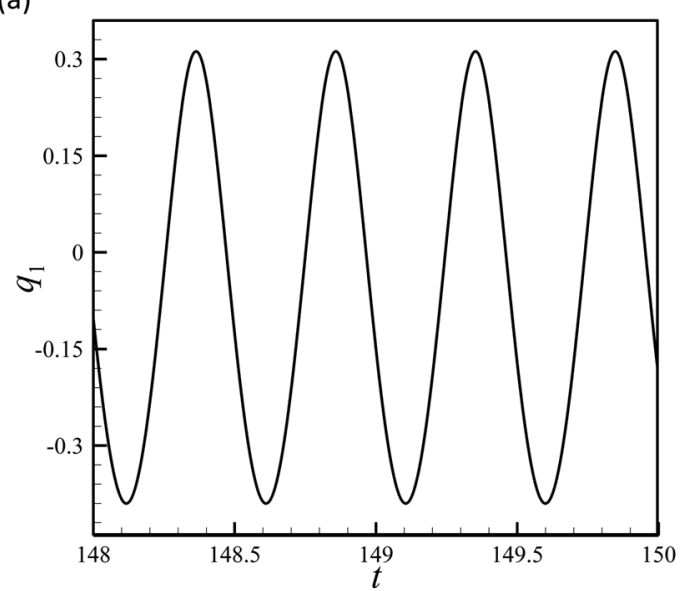

(b)

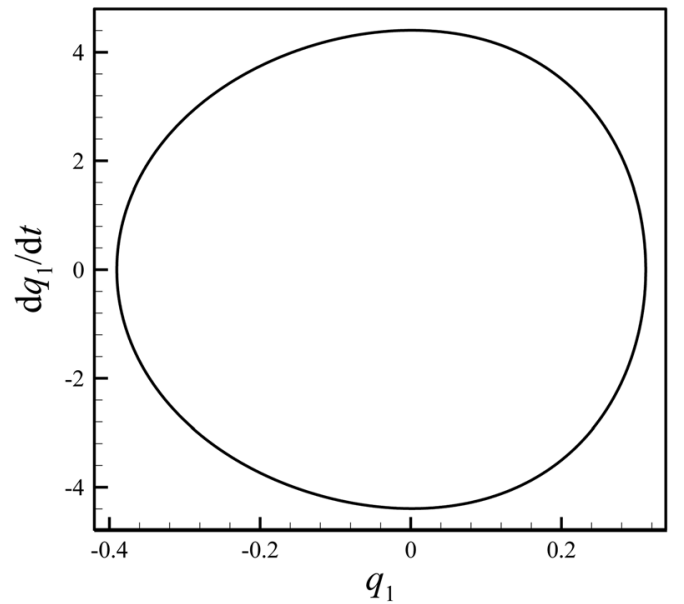

(c)

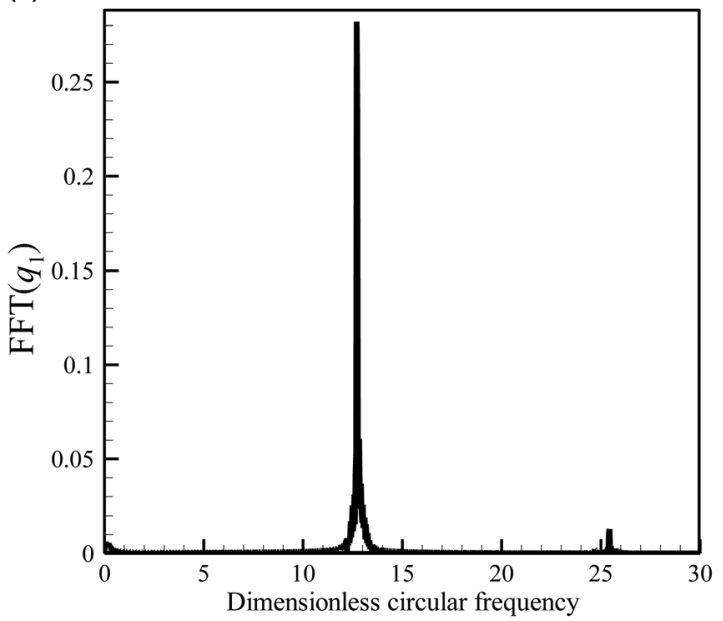

Fig. 3 The details of the dynamics of the system of Fig. 2 at $\Omega=1.0290 \omega_{1} ;(a)-(c)$ time trace, phase-plane portrait, and FFT of the $q_{1}$ motion, respectively.

The nonlinear coupled resonant responses of the system is shown in Fig. 8 in the form of force-response curves, obtained by varying the forcing amplitude $f_{1}$ where the excitation frequency is set to a fixed value $\left(\Omega=1.0650 \omega_{1}\right.$, here $) ; A_{0}=0.2$. There are two limit-point bifurcations present with one unstable solution branch in between. These two limit-point bifurcation occur at points $\mathrm{LP}_{1}$ and $\mathrm{LP}_{2}$, with $f_{1}=3.1647$ and $f_{1}=0.9769$, respectively. (a)

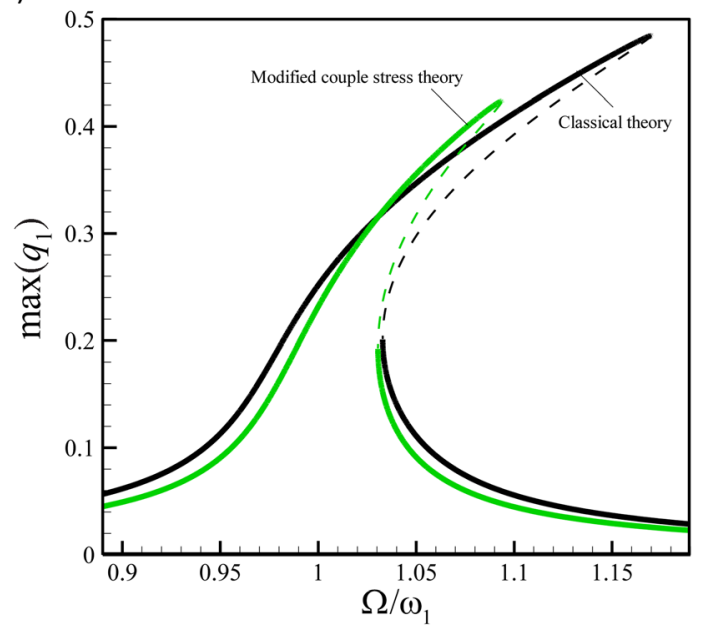

(b)

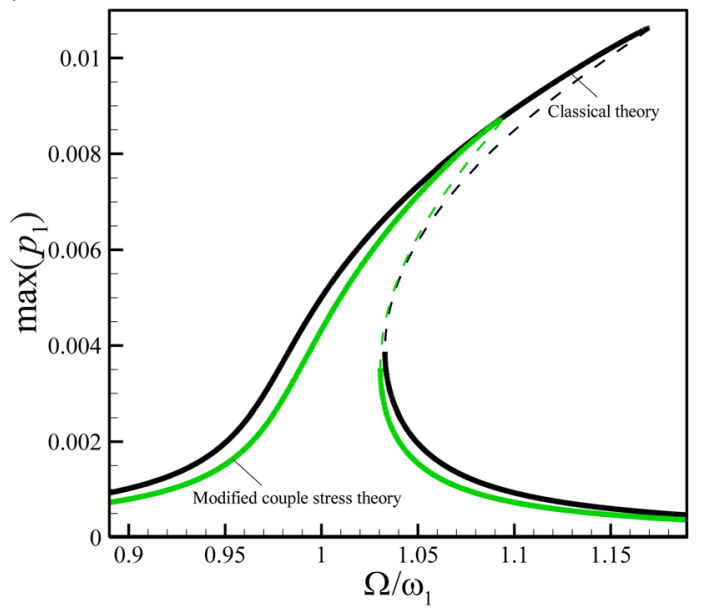

(c)

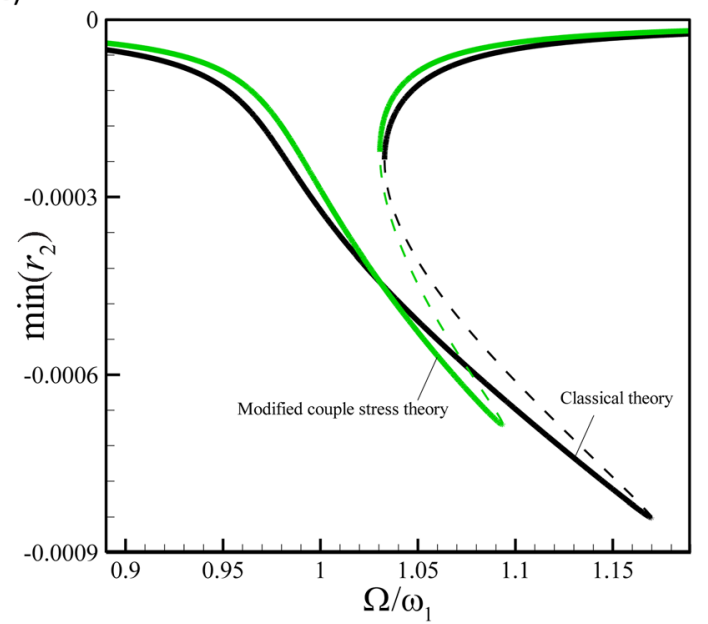

Fig. 4 Comparison between the frequency-response curves of the system obtained via the modified couple stress and classical theories: $(a),(b)$ the first generalized coordinate of the transverse motion and rotation, respectively; $(c)$ the second generalized coordinate of the longitudinal motion. $f_{1}=1.15$ and $A_{0}=0.2 ; I=17.6 \mu \mathrm{m}$ for the modified couple stress theory and $I=0$ for the classical theory. Solid and dashed lines represent the stable and unstable solutions, respectively.

The force-response curves for a larger initial amplitude of imperfection, i.e., $A_{0}=0.4$, are depicted in Fig. 9; the excitation frequency is set to $\Omega=0.9650 \omega_{1}$. It is seen that the number of limit-point bifurcations are increased to four, due to increased 
(a)

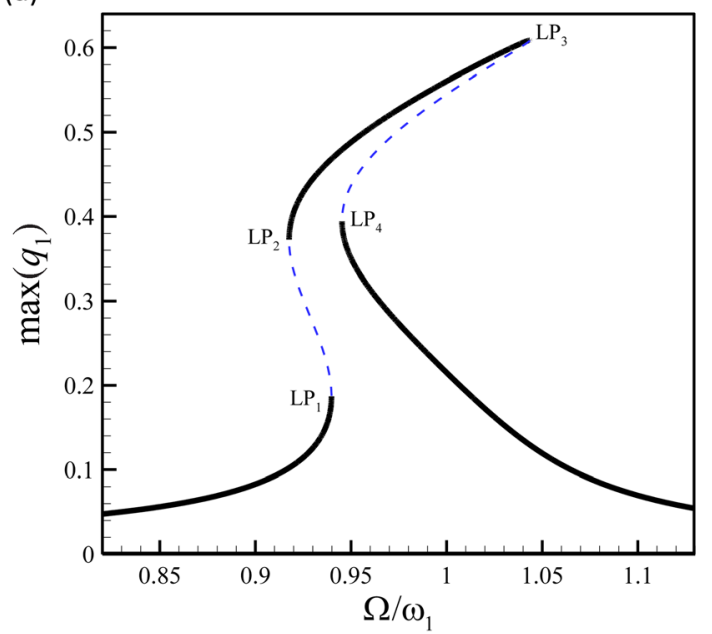

(b)

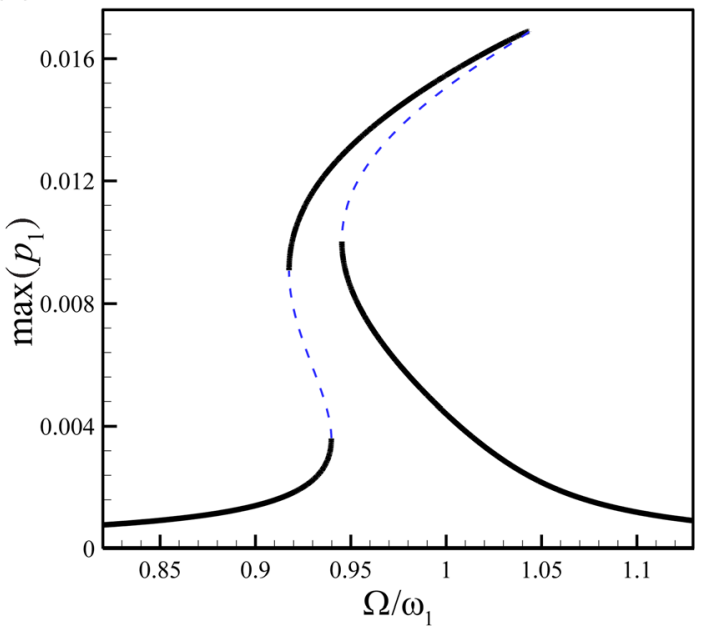

(c)

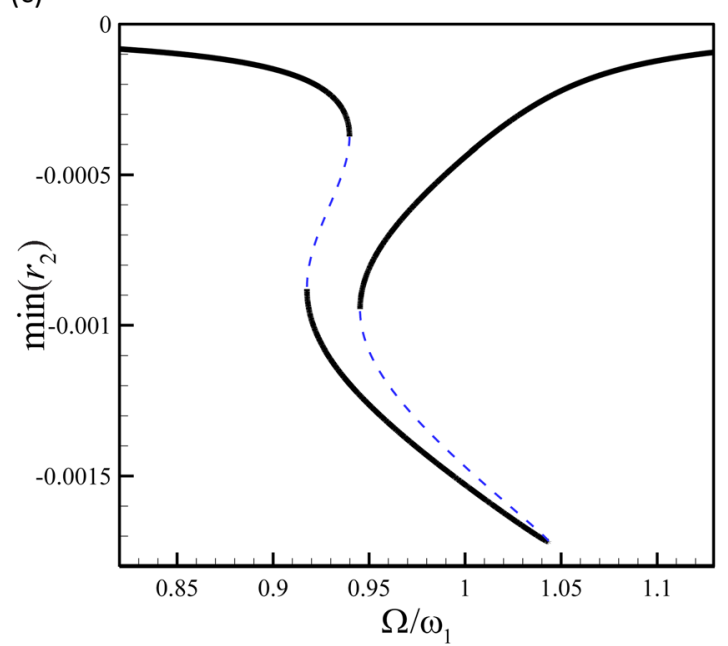

Fig. 5 Frequency-response curves of the system: $(a),(b)$ the first generalized coordinate of the transverse motion and rotation, respectively; (c) the second generalized coordinate of the longitudinal motion. Solid and dashed lines represent the stable and unstable solutions respectively; $f_{1}=2.85$ and $A_{0}=0.4$.

amplitude of initial imperfection. In particular, the limit-point bifurcations occur at $f_{1}=1.3082,0.8526,4.3612$, and 1.9584 , corresponding to points $\mathrm{LP}_{1}, \mathrm{LP}_{2}, \mathrm{LP}_{3}$, and $\mathrm{LP}_{4}$, respectively. It is seen that as $f_{1}$ is increased from 0.0, snap-through motions occur (a)

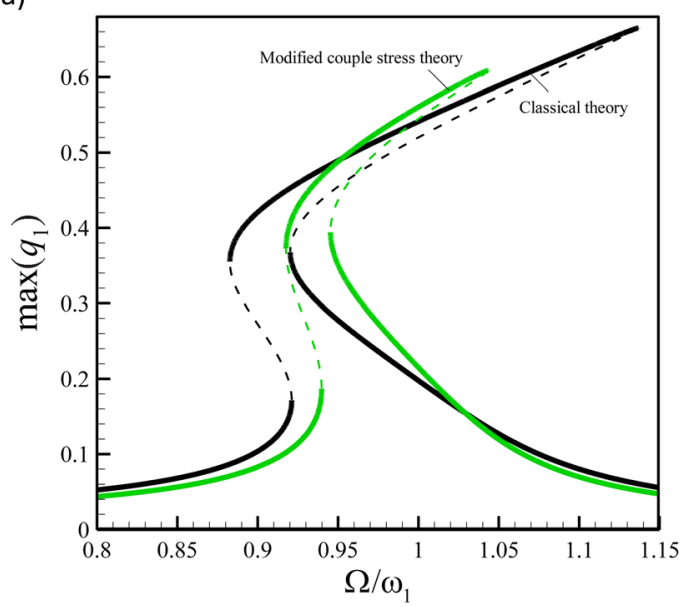

(b)

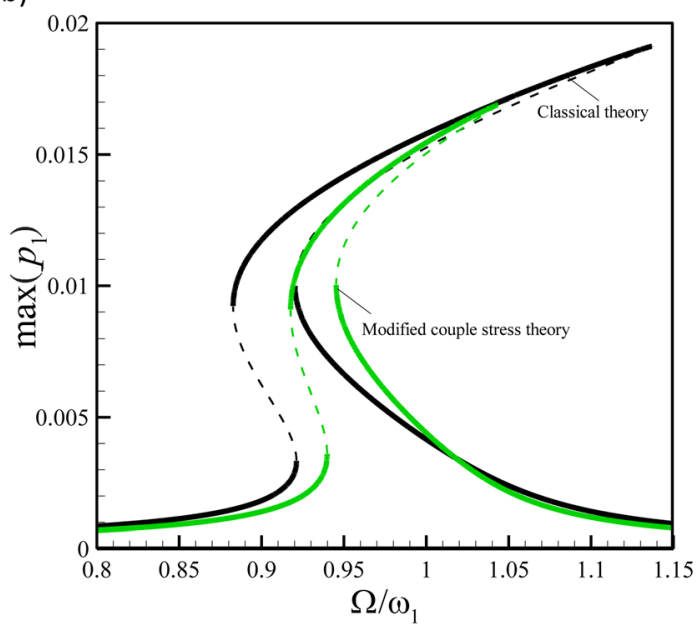

(c)

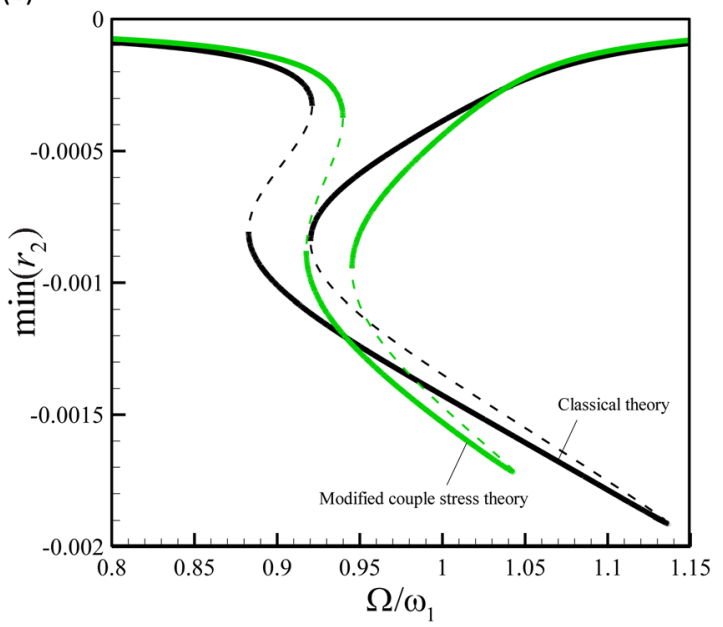

Fig. 6 Comparison between the frequency-response curves of the system obtained via the modified couple stress and classical theories: $(a),(b)$ the first generalized coordinate of the transverse motion and rotation, respectively; $(c)$ the second generalized coordinate of the longitudinal motion. $f_{1}=\mathbf{2 . 8 5}$ and $A_{0}=0.4 ; I=17.6 \mu \mathrm{m}$ for the modified couple stress theory and $I=0$ for the classical theory. Solid and dashed lines represent the stable and unstable solutions, respectively.

at points $\mathrm{LP}_{1}$ and $\mathrm{LP}_{3}$, where the motion becomes unstable and the system inevitably jumps to a higher-amplitude stable branch.

The force-response curves of the system for different values of the frequency ratio $\left(\Omega / \omega_{1}\right)$ are constructed and plotted together in 
Fig. 10 in order to analyze the effect of the frequency ratio on the coupled nonlinear resonant response of the system. It is seen in the subfigure (a) that as the frequency ratio is increased, the first two limit-point bifurcations occur at smaller forcing amplitudes while the third and fourth limit-point bifurcations take place at

(a)

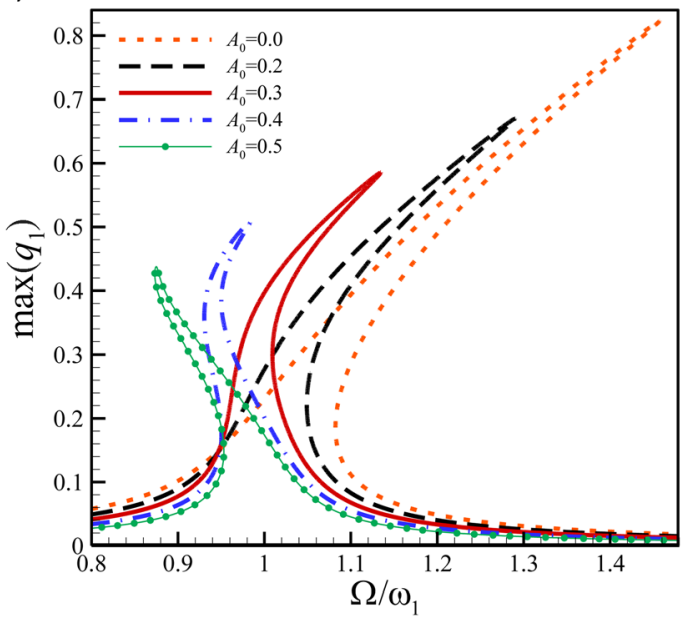

(b)

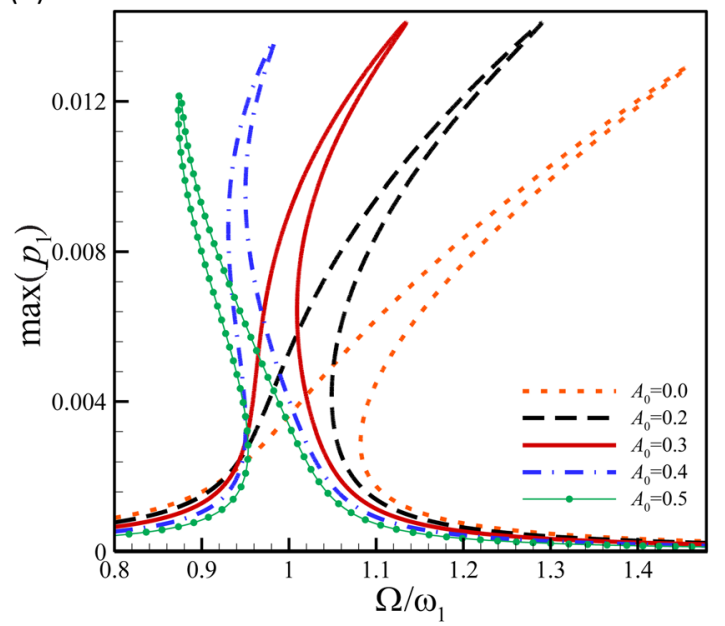

(c)

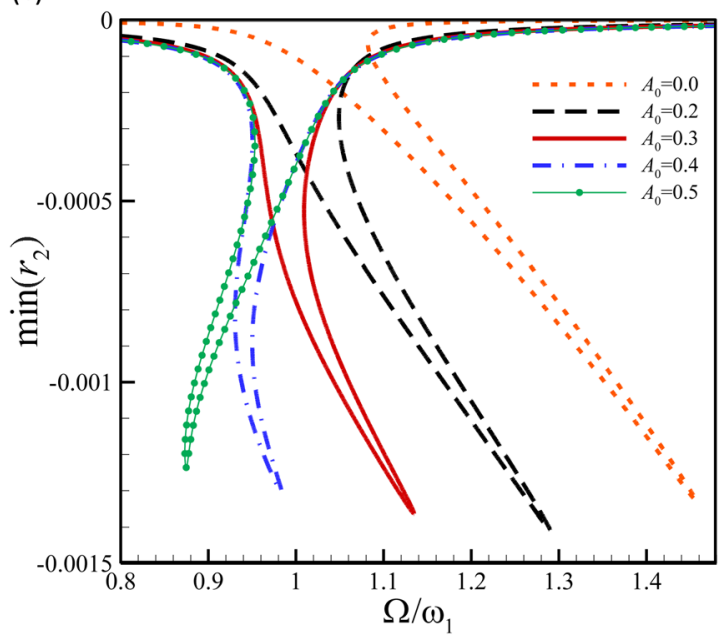

Fig. 7 Frequency-response curves of the system for several amplitudes of the initial imperfection, $A_{0}:(a),(b)$ the first generalized coordinate of the transverse motion and rotation, respectively; $(c)$ the second generalized coordinate of the longitudinal motion; $f_{1}=2.15$

Journal of Computational and Nonlinear Dynamics (a)

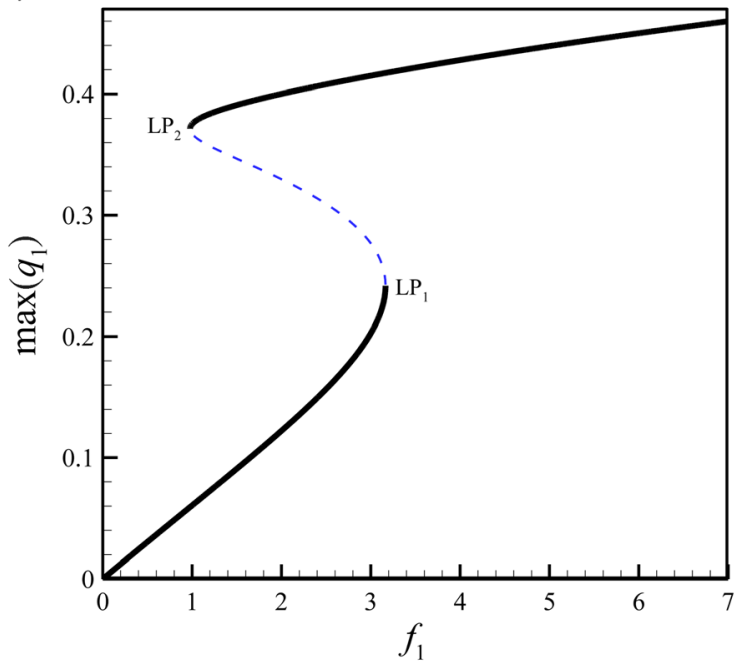

(b)

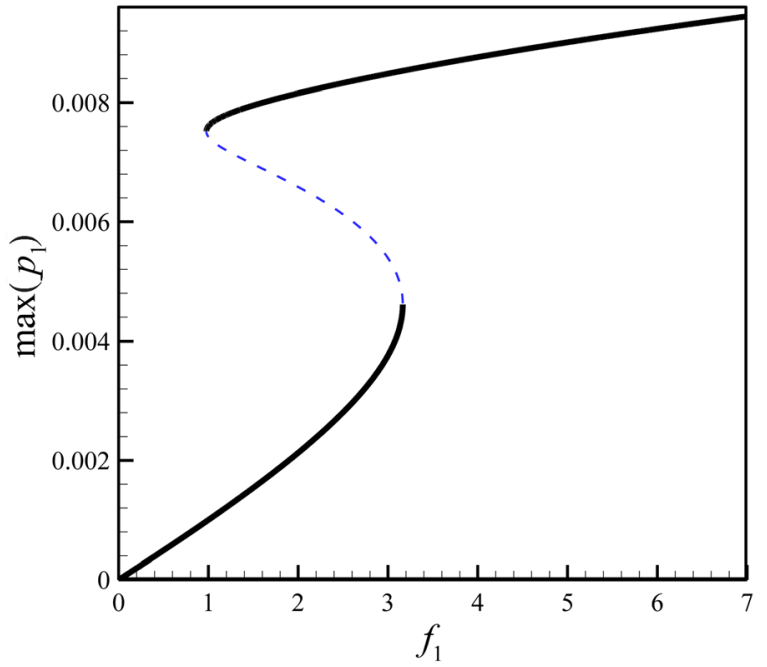

(c)

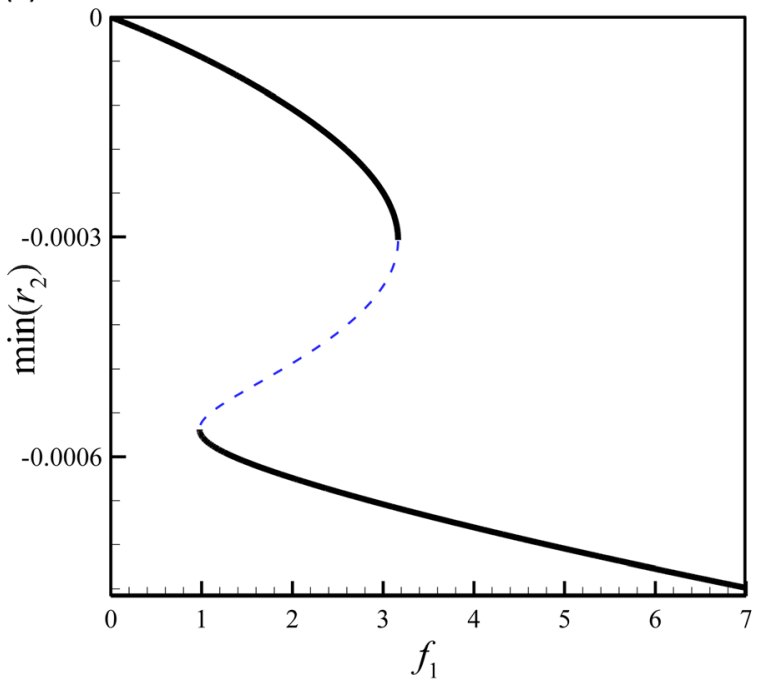

Fig. 8 Force-response curves of the system: (a), (b) the first generalized coordinate of the transverse motion and rotation, respectively; $(c)$ the second generalized coordinate of the longitudinal motion. Solid and dashed lines represent the stable and unstable solutions respectively; $\Omega=1.0650 \omega_{1}$ and $A_{0}=0.2$. 
larger excitation frequencies. Moreover, at sufficiently large forcing amplitudes, the amplitude of the oscillation increases with increasing $\Omega / \omega_{1}$.

Figure 11 highlights the effect of the amplitude of the initial imperfection on the force-response curves of the system. This figure illustrates that as the amplitude of the initial imperfection is increased, the limit-point bifurcations occur at larger forcing amplitudes; moreover, it is seen that for $A_{0}=0.5$, no limit-point bifurcation occurs for the ranges of the forcing amplitude studied here. (a)

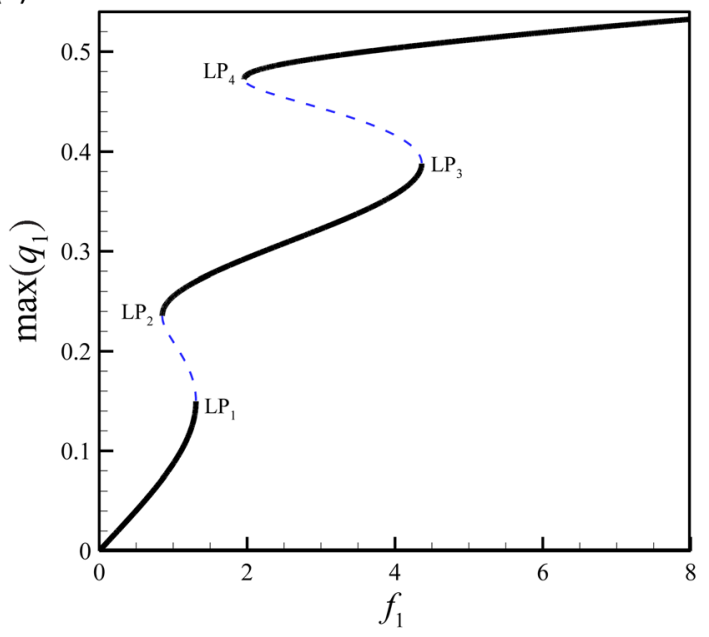

(b)

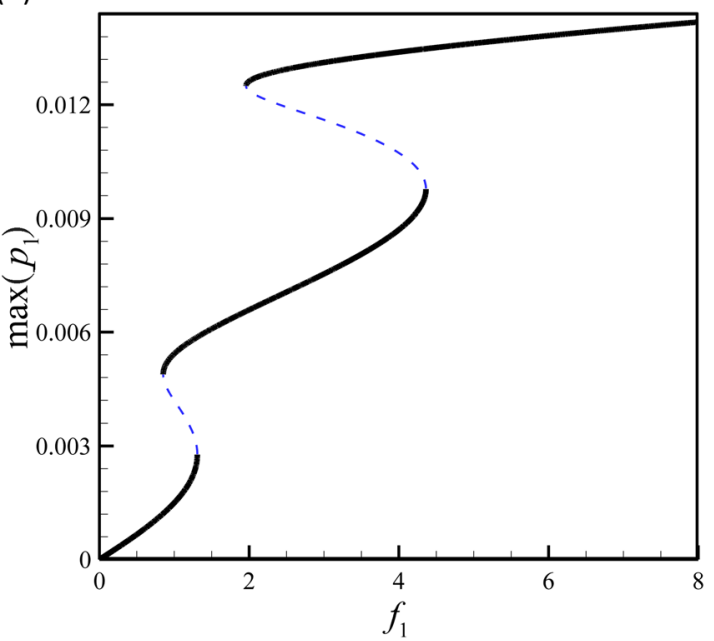

(c)

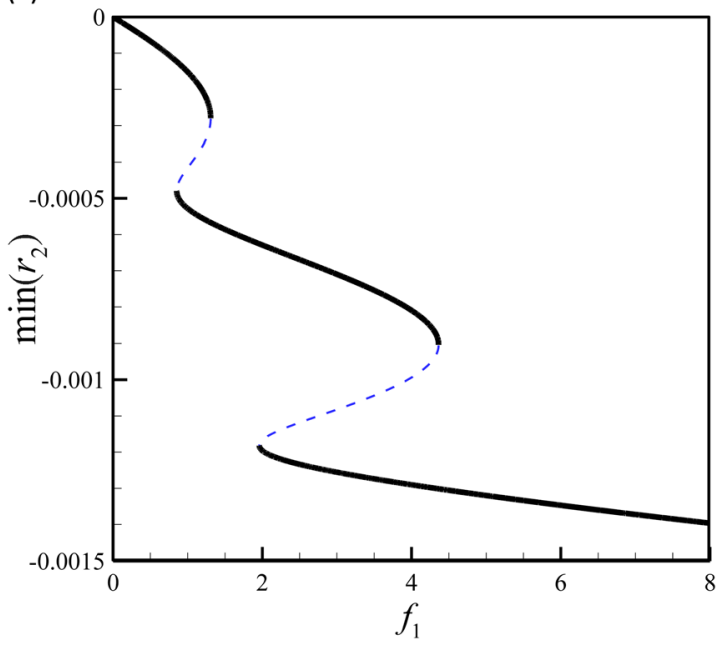

Fig. 9 Force-response curves of the system: $(a),(b)$ the first generalized coordinate of the transverse motion and rotation, respectively; $(c)$ the second generalized coordinate of the longitudinal motion. Solid and dashed lines represent the stable and unstable solutions respectively; $\Omega=0.9650 \omega_{1}$ and $A_{0}=0.4$.

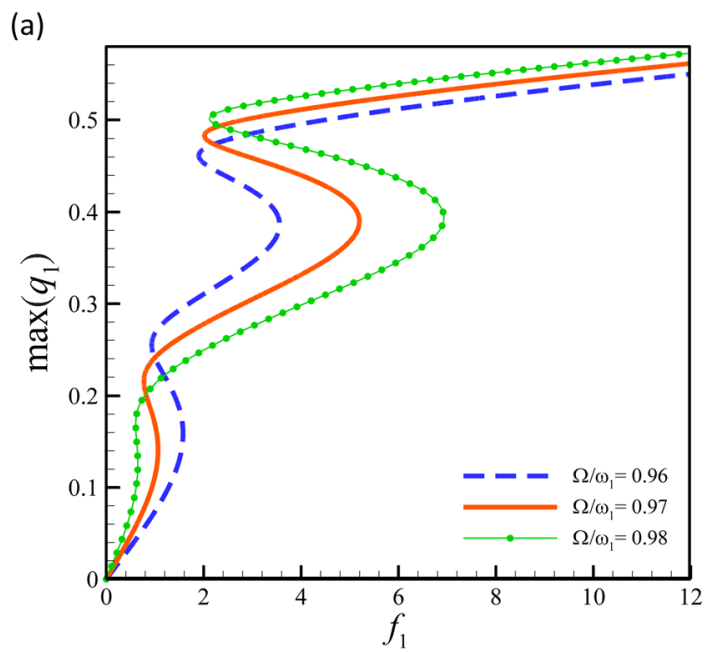

(b)

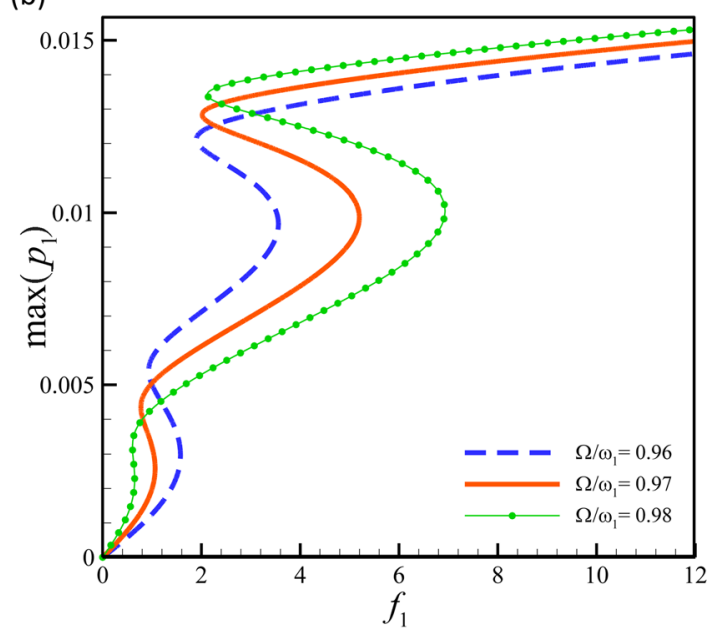

(c)

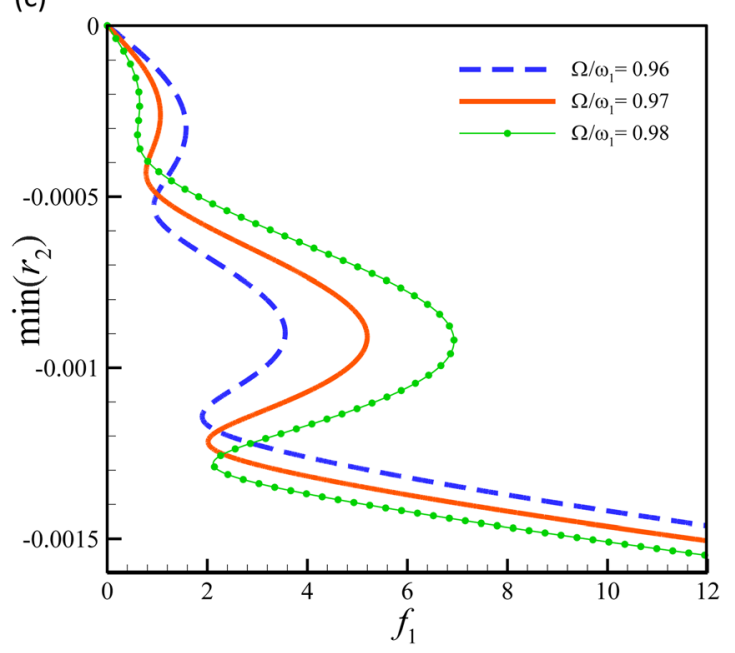

Fig. 10 Force-response curves of the system for different frequency ratios, $\Omega / \omega_{1}$ : (a), (b) the first generalized coordinate of the transverse motion and rotation, respectively; $(c)$ the second generalized coordinate of the longitudinal motion. $A_{0}=0.4$. 
(a)

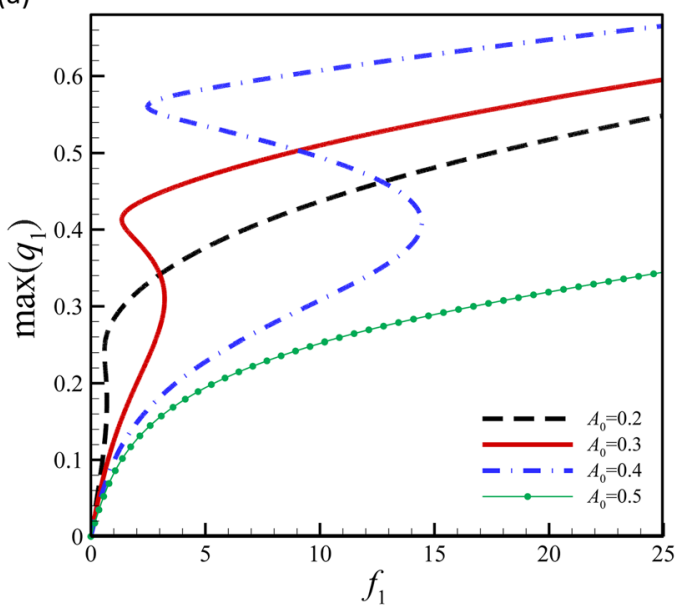

(b)

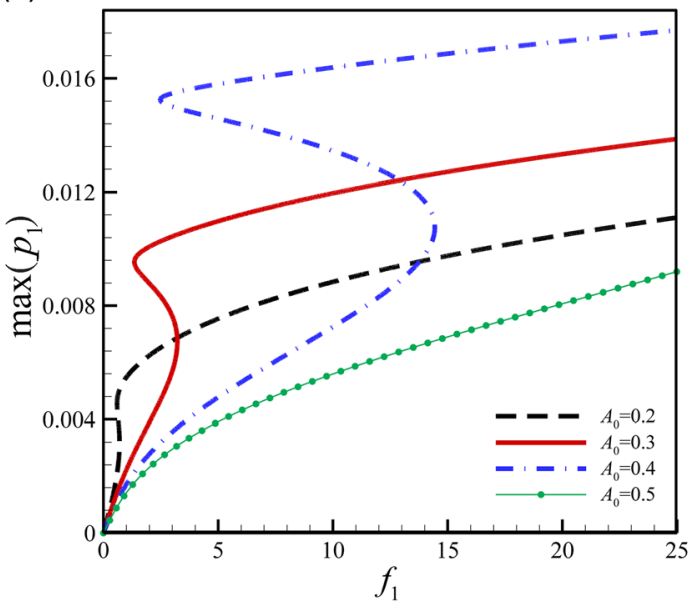

(c)

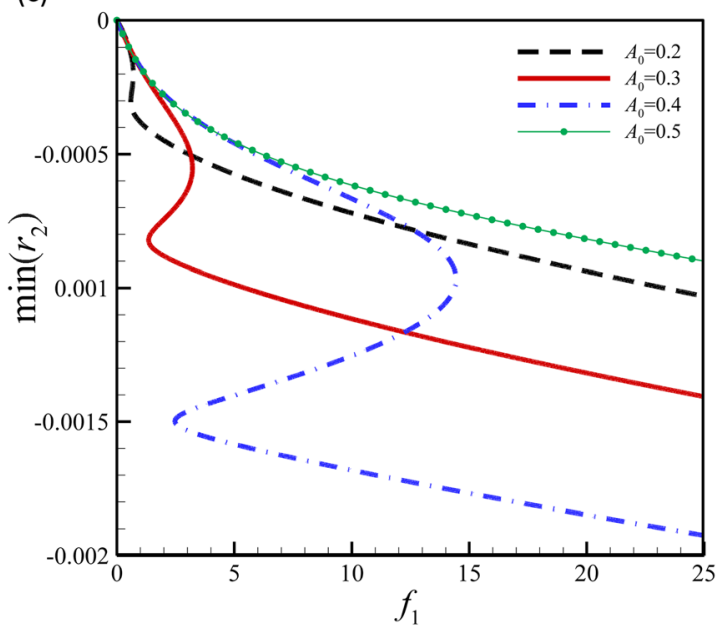

Fig. 11 Force-response curves of the system for several amplitudes of the initial imperfection, $A_{0}:(a),(b)$ the first generalized coordinate of the transverse motion and rotation, respectively; $(c)$ the second generalized coordinate of the longitudinal motion. $\Omega=1.02 \omega_{1}$.

\section{Concluding Remarks}

The coupled nonlinear dynamics of a geometrically imperfect third-order shear deformable extensible microbeam has been analyzed in this paper numerically based on the modified couple stress theory. The model development became possible through use of Hamilton's principle along with constitutive relations. The initial imperfection was modeled in the form of a trigonometric distribution of the deflection along the microbeam length. Galerkin's method was employed to obtain a high-dimensional discretized model. This model was solved by means of the pseudo-arclength continuation technique and direct timeintegration based on the Rosenbrock method.

Analyzing the frequency-response curves of the system showed that the system displays only a hardening-type behavior for smaller amplitudes of the initial imperfection-for higher amplitudes of the initial imperfection, on the other hand, both softening and hardening behaviors are displayed by the system; due to presence of the initial imperfection, the system displays asymmetric oscillations; the modified couple stress theory predicts smaller amplitudes of oscillation as well as weaker hardening and softening nonlinear behaviors; as the amplitude of the initial imperfection is increased, the initial softening behavior becomes stronger.

Examining the force-response curves of the system revealed that the system displays two limit-point bifurcations for smaller amplitudes of the initial imperfection and four limit-point bifurcations for sufficiently large amplitudes of initial imperfection; increasing the excitation frequency hasten the occurrences of the first two limit-point bifurcations while delaying that of the third and fourth limit-point bifurcations; as a result of increased amplitude of the initial imperfection, the limit-point bifurcations (if any) occur at higher forcing amplitudes.

\section{Acknowledgment}

The financial support to this research by the start-up grant of the University of Wollongong is gratefully acknowledged.

\section{References}

[1] Ouakad, H. M., 2013, "An Electrostatically Actuated MEMS Arch Band-Pass Filter," Shock Vib., 20(4), pp. 809-819.

[2] Wang, W., and Soper, S. A., 2006, Bio-MEMS: Technologies and Applications, Taylor \& Francis, Boca Raton, FL.

[3] Abdel-Rahman, E. M., Younis, M. I., and Nayfeh, A. H., 2002 , "Characterization of the Mechanical Behavior of an Electrically Actuated Microbeam,” J. Micromech. Microeng., 12(6), pp. 759-766.

[4] Younis, M. I., Abdel-Rahman, E. M., and Nayfeh, A., 2003, "A Reduced-Order Model for Electrically Actuated Microbeam-Based MEMS," J. Microelectromech. Syst., 12(5), pp. 672-680.

[5] Younis, M. I., and Nayfeh, A. H., 2003, "A Study of the Nonlinear Response of a Resonant Microbeam to an Electric Actuation," Nonlinear Dyn., 31(1), pp. 91-117.

[6] Nayfeh, A. H., and Younis, M. I., 2005, "Dynamics of MEMS Resonators Under Superharmonic and Subharmonic Excitations," J. Micromech. Microeng., 15(10), pp. 1840-1847.

[7] Ouakad, H. M., and Younis, M. I., 2010, "The Dynamic Behavior of MEMS Arch Resonators Actuated Electrically,” Int. J. Non Linear Mech., 45(7), pp. 704-713.

[8] Younis, M. I., Ouakad, H. M., Alsaleem, F. M., Miles, R., and Cui, W., 2010 "Nonlinear Dynamics of MEMS Arches Under Harmonic Electrostatic Actuation," Microelectromech. Syst., 19(3), pp. 647-656.

[9] McFarland, A. W., and Colton, J. S., 2005, "Role of Material Microstructure in Plate Stiffness With Relevance to Microcantilever Sensors," J. Micromech. Microeng., 15(5), p. 1060

[10] Fleck, N. A., Muller, G. M., Ashby, M. F., and Hutchinson, J. W., 1994, "Strain Gradient Plasticity: Theory and Experiment," Acta Metall. Mater., 42(2) pp. 475-487.

[11] Lam, D. C. C., Yang, F., Chong, A. C. M., Wang, J., and Tong, P., 2003, "Experiments and Theory in Strain Gradient Elasticity," J. Mech. Phys. Solids, 51(8), pp. 1477-1508.

[12] Farokhi, H., and Ghayesh, M., 2015, "Size-Dependent Behaviour of Electrically Actuated Microcantilever-Based MEMS," Int. J. Mech. Mater. Des. (in press).

[13] Kong, S., Zhou, S., Nie, Z., and Wang, K., 2008, "The Size-Dependent Natural Frequency of Bernoulli-Euler Micro-Beams," Int. J. Eng. Sci., 46(5), pp. 427-437.

[14] Ma, H. M., Gao, X. L., and Reddy, J. N., 2008, “A Microstructure-Dependent Timoshenko Beam Model Based on a Modified Couple Stress Theory," J. Mech. Phys. Solids, 56(12), pp. 3379-3391.

[15] Asghari, M., Kahrobaiyan, M., Rahaeifard, M., and Ahmadian, M., 2011, "Investigation of the Size Effects in Timoshenko Beams Based on the Couple Stress Theory," Arch. Appl. Mech., 81(7), pp. 863-874.

[16] Wang, B., Zhao, J., and Zhou, S., 2010, "A Micro Scale Timoshenko Beam Model Based on Strain Gradient Elasticity Theory," Eur. J. Mech. A. Solids, 29(4), pp. 591-599.

[17] Ansari, R., Faghih Shojaei, M., Gholami, R., Mohammadi, V., and Darabi, M. A., 2013, "Thermal Postbuckling Behavior of Size-Dependent Functionally Graded Timoshenko Microbeams,” Int. J. Non Linear Mech., 50, pp. 127-135. 
[18] Ansari, R., Gholami, R., Faghih Shojaei, M., Mohammadi, V., and Sahmani, S., 2013, "Size-Dependent Bending, Buckling and Free Vibration of Functionally Graded Timoshenko Microbeams Based on the Most General Strain Gradient Theory," Compos. Struct., 100, pp. 385-397.

[19] Ansari, R, Gholami, R., and Sahmani, S., 2011, "Free Vibration Analysis of Size-Dependent Functionally Graded Microbeams Based on the Strain Gradient Timoshenko Beam Theory,” Compos. Struct., 94(1), pp. 221-228.

[20] Nateghi, A., Salamat-Talab, M., Rezapour, J., and Daneshian, B., 2012, "Size Dependent Buckling Analysis of Functionally Graded Micro Beams Based on Modified Couple Stress Theory," Appl. Math. Modell., 36(10), pp. 4971-4987.

[21] Akgöz, B., and Civalek, Ö., 2012, "Analysis of Micro-Sized Beams for Various Boundary Conditions Based on the Strain Gradient Elasticity Theory," Arch. Appl. Mech., 82(3), pp. 423-443.

[22] Ouakad, H. M., and Younis, M. I., 2014, "On Using the Dynamic SnapThrough Motion of MEMS Initially Curved Microbeams for Filtering Applications," J. Sound Vib., 333(2), pp. 555-568.

[23] Farokhi, H., Ghayesh, M., and Amabili, M., 2013, "Nonlinear Resonant Behavior of Microbeams Over the Buckled State," Appl. Phys. A, 113(2), pp. 297-307.

[24] Ramezani, S., 2012, "A Micro Scale Geometrically Non-Linear Timoshenko Beam Model Based on Strain Gradient Elasticity Theory," Int. J. Non Linear Mech., 47(8), pp. 863-873.

[25] Asghari, M., Kahrobaiyan, M. H., and Ahmadian, M. T., 2010, "A Nonlinear Timoshenko Beam Formulation Based on the Modified Couple Stress Theory," Int. J. Eng. Sci., 48(12), pp. 1749-1761.

[26] Salamat-talab, M., Nateghi, A., and Torabi, J., 2012, "Static and Dynamic Analysis of Third-Order Shear Deformation FG Micro Beam Based on Modified Couple Stress Theory,” Int. J. Mech. Sci., 57(1), pp. 63-73.

[27] Simsek, M., and Reddy, J. N., 2013, "Bending and Vibration of Functionally Graded Microbeams Using a New Higher Order Beam Theory and the Modified Couple Stress Theory," Int. J. Eng. Sci., 64, pp. 37-53.
[28] Simsek, M., and Reddy, J. N., 2013, “A Unified Higher Order Beam Theory for Buckling of a Functionally Graded Microbeam Embedded in Elastic Medium Using Modified Couple Stress Theory," Compos. Struct., 101, pp. 47-58.

[29] Sahmani, S., and Ansari, R., 2013, "Size-Dependent Buckling Analysis of Functionally Graded Third-Order Shear Deformable Microbeams Including Thermal Environment Effect," Appl. Math. Modell., 37(23), pp. 9499-9515.

[30] Zhang, B., He, Y. M., Liu, D. B., Gan, Z. P., and Shen, L., 2014, "SizeDependent Functionally Graded Beam Model Based on an Improved ThirdOrder Shear Deformation Theory," Eur. J. Mech. A-Solid, 47, pp. 211-230.

[31] Ghayesh, M., Farokhi, H., and Amabili, M., 2013, "Coupled Nonlinear SizeDependent Behaviour of Microbeams," Appl. Phys. A, 112(2), pp. 329-338.

[32] Ghayesh, M. H., Farokhi, H., and Amabili, M., 2014, "In-Plane and Out-ofPlane Motion Characteristics of Microbeams With Modal Interactions," Compos. Part B: Eng., 60, pp. 423-439.

[33] Gholipour, A., Farokhi, H., and Ghayesh, M., 2014, "In-Plane and Out-OfPlane Nonlinear Size-Dependent Dynamics of Microplates," Nonlinear Dyn., 79(3), pp. 1771-1785.

[34] Farokhi, H., and Ghayesh, M. H., 2015, "Nonlinear Dynamical Behaviour of Geometrically Imperfect Microplates Based on Modified Couple Stress Theory,” Int. J. Mech. Sci., 90, pp. 133-144.

[35] Ghayesh, M. H., and Farokhi, H., 2015, "Nonlinear Dynamics of Microplates," Int. J. Eng. Sci., 86, pp. 60-73.

[36] Ghayesh, M. H., Amabili, M., and Farokhi, H., 2013, "Three-Dimensional Nonlinear Size-Dependent Behaviour of Timoshenko Microbeams," Int. J. Eng. Sci., 71, pp. 1-14.

[37] Benedettini, F., and Rega, G., 1987, "Non-Linear Dynamics of an Elastic Cable Under Planar Excitation,” Int. J. Non Linear Mech., 22(6), pp. 497-509.

[38] Rega, G., and Benedettini, F., 1989, "Planar Non-Linear Oscillations of Elastic Cables Under Subharmonic Resonance Conditions," J. Sound Vib., 132(3), pp. 367-381. 